\title{
Einfluss des therapeutischen Qi auf die Herzratenvariabilität bei Akupunktur: Eine randomisierte kontrollierte Studie
}

\author{
Raphael J. Hochstrasser ${ }^{a, b}$ \\ P. Christian Endler ${ }^{a}$ \\ Sabine \\ a Interuniversitäres Kolleg, Schloss Seggau, Graz, Österreich; \\ bTCM Aarau, Praxiszentrum für Chinesische Medizin, Aarau, Schweiz; \\ cInstitut für Komplementärmedizin, Universität Bern, Bern, Schweiz; \\ dArbeitsgruppe Forschung, Schweizerische Berufsorganisation für Traditionelle \\ Chinesische Medizin SBO-TCM, Degersheim, Schweiz
}

Dlein ${ }^{\mathrm{c}, \mathrm{d}}$

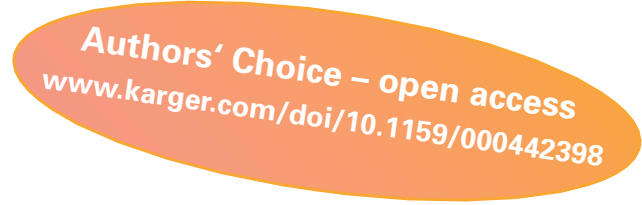

\section{Schlüsselwörter}

Akupunktur · Herzratenvariabilität · Neiguan .

Akupunkturmechanismus - Qi - Autonomes Nervensystem . Herzfrequenz - Randomisierte kontrollierte Studie ·

Traditionelle Chinesische Medizin · Qi Gong

\section{Zusammenfassung}

Hintergrund: In einer kürzlich durchgeführten Studie konnten Probanden bei der Akupunktur eine Stimulation mittels "therapeutischem Qi» wahrnehmen, obwohl mechanische und psychologische Faktoren ausgeschlossen wurden. Nun untersuchten wir, ob das therapeutische Qi auch die Herzratenvariabilität messbar beeinflusst. Methoden: Es handelte sich um eine randomisierte, kontrollierte, einfach verblindete Crossover-Studie mit 30 Probanden. Mithilfe einer Vorrichtung wurde eine Akupunkturnadel in den Akupunkt Pe6 eingeführt. Mit einer weiteren Vorrichtung wurde der herausragende Teil der Nadel so fixiert, dass bei Berührung des Nadelendes die Übertragung von mechanischen Bewegungen auf die Nadelspitze ausgeschlossen werden konnte. Bei der anschließenden Prüfintervention wurde das Nadelende zur Stimulation des Qi durch den Therapeuten berührt, bei der Kontrollintervention wurde die Nadel nicht berührt, sodass das Qi nicht stimuliert wurde. Bei der Nullintervention wurde keine Nadel gesetzt. Bei jeder Intervention wurden jeweils vorher und nachher Parameter der Herzratenvariabilität (HRV) ermittelt. Ergebnisse: Zwischen den drei Interventionen konnten keine signifikanten Unterschiede der HRV-Parameter festgestellt werden. Zwischen Baselineund Endmessung sank die Herzfrequenz bei der Kontrollund Prüfintervention ( $-2 \mathrm{~min}^{-1}, \mathrm{~d}=0,24$ bzw. $-2 \mathrm{~min}^{-1}, \mathrm{~d}=$ $0,24)$, die RMSSD stieg mit der Nadelberührung bzw. der Stimulation des Qi signifikant an $(+9.7 \mathrm{~ms}, \mathrm{~d}=-0.32)$. Schlussfolgerung: Die zuvor subjektiv wahrgenommene Stimulation mit therapeutischem Qi konnte innerhalb dieses Settings anhand von HRV-Parametern nicht ausreichend bestätigt werden.

\section{Keywords}

Acupuncture $\cdot$ Heart rate variability $\cdot$ Neiguan .

Acupuncture mechanism - Qi · Autonomic nervous system . Heart rate $\cdot$ Randomized controlled trial .

Traditional Chinese Medicine · Oigong

\section{Summary}

Influence of Therapeutic Qi on Heart Rate Variability in

Acupuncture: a Randomized Controlled Study

Background: In a recent acupuncture study, volunteers were able to sense stimulation by 'therapeutic Qi' even when mechanical and psychological causes were excluded. Here, we investigated if 'therapeutic $\mathrm{Qi}^{\prime}$ also influences the heart rate variability. Methods: This was a controlled, randomized, single-blind crossover study with 30 volunteers. Using a special device, an acupuncture needle was inserted in the acupuncture point Pe6. With another device, the protruding end of the needle was fixed so that no transmission of mechanical movement to the tip was possible when the handle was touched. During the experimental intervention, the therapist touched the handle of the needle to stimulate Qi. During the control intervention, the needle was left untouched. During the null intervention, no needle was inserted. Before and after the interventions, parameters of heart rate variability (HRV) were measured. Results: Between the 3 interventions no significant differences in HRV parameters were observed. From pre- to post-measurement the heart rate decreased in the control and experimental interventions $(-2$ $\min ^{-1}, d=0.24$ and $-2 \min ^{-1}, d=0.24$, respectively), RMSSD increased significantly when the needle was touched and Qi was stimulated $(+9.7 \mathrm{~ms}, \mathrm{~d}=-0.32)$. Conclusion: Within this setting the subjectively perceived stimulation with therapeutic Qi could not be confirmed by using objective HRV measures. 


\section{Einführung}

Für die Akupunkturforschung gewann die Analyse der Herzratenvariabilität (HRV) in den letzten Jahren zunehmend an Bedeutung [1]. Das menschliche Herz schlägt in einem variablen Rhythmus, der primär vom vegetativen Nervensystem vorgegeben wird. Während die parasympathische Aktivierung die Herzschlagfrequenz verringert, erhöht die sympathische Aktivierung die Herzschlagfrequenz [2]. Das ist vergleichbar mit Yin und Yang (chin: Yīn Yáng, 阴阳), den Grundprinzipien der Chinesischen Medizin [3]. Der Yin-Aspekt verkörpert die Ressourcen und das Potenzial, welche durch Erholung und Regeneration im parasympathischen Zustand konsolidiert werden; der Yang-Aspekt entspricht der Leistungskraft, die durch die sympathische Aktivierung mobilisiert wird. Der weiße Punkt im schwarzen Teil der Yin-Yang-Darstellung, das sogenannte Yang im Yin, könnte mit der parasympathischen Modulation verglichen werden, welche schnell und hochfrequent $(0,15-0,40 \mathrm{~Hz})$ erfolgt, während die sympathische Modulation, das Yin im Yang, im vergleichsweise niederfrequenten $(0,04-$ $0115 \mathrm{~Hz}$ ) Bereich liegt.

Gemäß dem naturphilosophischen Verständnis der Chinesischen Medizin basiert die Wirkung von Akupunktur auf Qi (chin: Qì, 气), welches in einem körperweit verzweigten Netzwerk fließt und insbesondere an den Akupunkten mittels Nadeln reguliert werden kann [4]. Qi wird als die universale Energie betrachtet, aus der alles Existierende besteht, sowohl stoffliche, wie auch nichtstoffliche Aspekte [3-5].

Das Praktizieren von Übungen, welche die Qualität und Dynamik von Qi positiv beeinflussen sollen, wird Qi Gong genannt (chin: Qì Gōng, 气功, deutsch: 'Energie-Arbeit') [6]. Der positive Einfluss von Qi Gong wurde einerseits bei den Praktizierenden selbst beobachtet [7, 8], andererseits führte durch das Qi Gong hervorgebrachte externe Qi bei verschiedenen Krebszelllinien zur Apoptose [9-11]. Dementsprechend wird bei Anwendung von Akupunktur in der Chinesischen Medizin versucht, das Qi des Patienten positiv zu beeinflussen [12]. Wir gehen davon aus, dass - ähnlich wie beim Qi Gong - das häufige Praktizieren von Akupunktur zu einer Art von Konditionierung der Effekte führen könnte.
In einer kürzlich von uns durchgeführten Studie konnten Probanden die Stimulation einer Akupunkturnadel mittels therapeutischem Qi subjektiv wahrnehmen, obwohl mechanische und psychologische Faktoren ausgeschlossen wurden [13]. Das Ziel der vorliegenden Studie war nun zu untersuchen, ob das therapeutische Qi auch einen Einfluss auf objektiv messbare Parameter wie die HRV hat. Dazu wählten wir den Akupunkturpunkt Pe6, dessen Einfluss auf das vegetative Nervensystem bereits bekannt ist [14].

\section{Methoden}

\section{Studiendesign}

Bei dieser Untersuchung handelt es sich um eine experimentelle, randomisierte, kontrollierte, einfach verblindete Crossover-Studie mit 30 Probanden. Die Anzahl von 30 Probanden wurde aufgrund unserer Vorstudie [13] und anderer vergleichbarer Studien $[15,16]$ gewählt.

Versuchsaufbau und Hilfsmittel

Zur Verblindung wurden verschiedene Vorkehrungen getroffen [17]. Zunächst streckte der liegende Proband seinen rechten Unterarm durch einen Sichtschutz hindurch und legte ihn in den Veliusator. Mit dem Akuplikator wurde dort eine Akupunkturnadel in den Akupunkturpunkt Pe6 (chin: Nèi Guān, 内关) insertiert (Abb. 1a). Der Akuplikator erlaubt die standardisierte Nadelinsertion mit gleichbleibender Kraft bzw. Einstichtiefe (durchschnittlich 12,3 $\pm 1,5 \mathrm{~mm}$ [17]) und ohne, dass die Nadel von der Prüfperson berührt werden muss. Danach wurde der herausragende Teil der Nadel im Veliusator von zwei Klemmbacken fixiert (Abb. 1b). In der Prüfphase wurde das Nadelende zwar vom Akupunkteur berührt, der Veliusator verhinderte jedoch die Übertragung von mechanischen Bewegungen auf die Nadelspitze (Abb. 1c).

\section{Versuchsablauf}

Der Versuchsablauf wird in Abbildung 2 auf einer Zeitachse dargestellt. Zunächst wurde jeder Proband über den Ablauf instruiert und gebeten, sich auf die Behandlungsliege zu legen. Auf die Probemessung mit dem HRV-Gerät folgten eine Pause und dann die Baselinemessung. Danach wurde eine der drei 10-minutigen Interventionen durch Würfeln bestimmt und durchgeführt. Bei der Nullintervention (A) verweilten die Probanden liegend in einer Ruhephase ohne weitere Intervention. Bei der Kontrollintervention (B) wurde die Akupunkturnadel zwar insertiert und fixiert, jedoch vom Therapeuten nicht berührt und somit auch nicht energetisch stimuliert. Bei der Prüfinter-
Abb. 1. Die zur Verblindung verwendeten Vorrichtungen Akuplikator und Veliusator.

Abb. 2. Zeitachse des Versuchsablaufs.
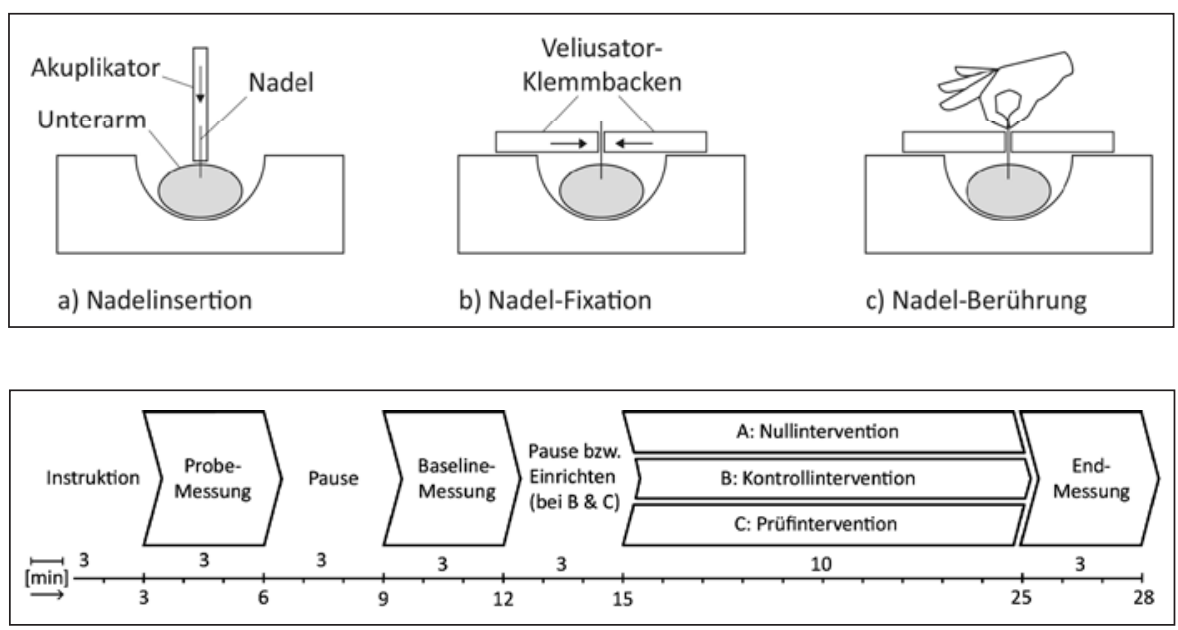
Tab. 1. Definition der verwendeten Parameter

\begin{tabular}{|c|c|c|}
\hline Parameter & Definition & Bedeutung/Normalwerte \\
\hline Bpm & $\begin{array}{l}\text { durchschnittliche Herzschlagfrequenz pro } \\
\text { Minute }\end{array}$ & \\
\hline $\mathrm{LF}, \mathrm{ms}^{2}$ & $\begin{array}{l}\text { niedrige Frequenzanteile der Variabilität } \\
(0,04-0,15 \mathrm{~Hz})[18]\end{array}$ & $\begin{array}{l}\text { frequenzanalytisches Maß für die hauptsächlich sympathische } \\
\text { Aktivierung [19] } \\
\text { Normalbereich: 754-1586 [18] }\end{array}$ \\
\hline $\mathrm{HF}, \mathrm{ms}^{2}$ & $\begin{array}{l}\text { hohe Frequenzanteile der Variabilität } \\
(0,15-0,4 \mathrm{~Hz})[18]\end{array}$ & $\begin{array}{l}\text { frequenzanalytisches, selektives Maß der parasympathischen } \\
\text { Aktivität [19] } \\
\text { Normalbereich: } 772-1178[18]\end{array}$ \\
\hline LF:HF & Verhältnis von LF zu HF & $\begin{array}{l}\text { Balance bzw. relativer Ausprägungsgrad der sympathischen } \\
\text { gegenüber der parasympathischen Aktivierung [19] } \\
\text { Normalbereich: } 1,5-2,0[18]\end{array}$ \\
\hline SDNN, ms & Standardabweichung aller RR-Intervalle [18] & $\begin{array}{l}\text { zeitanalytisches Maß für das Gesamtregulationsniveau [19] } \\
\text { Normalbereich: } 102-180 \text { [18] }\end{array}$ \\
\hline RMSSD, ms & $\begin{array}{l}\text { quadratischer Mittelwert der Summe aller } \\
\text { Differenzen benachbarter RR-Intervalle [18] }\end{array}$ & $\begin{array}{l}\text { zeitanalytisches Maß der parasympathischen } \\
\text { Herzschlagregulation [20] } \\
\text { Normalbereich: } 15-39[18]\end{array}$ \\
\hline
\end{tabular}

vention (C) wurde die Akupunkturnadel insertiert, fixiert, und das aus dem Veliusator herausragende Nadelende wurde vom Therapeuten zur Stimulation des Qi berührt. Nach der jeweiligen Interventionsphase wurde die Nadel entnommen und die Endmessung durchgeführt. Die drei Interventionen wurden bei identischem Setting mit jedem Probanden an jeweils verschiedenen Tagen zwischen März und Dezember 2013 in den Behandlungsräumen des Tao Chi, Schweizerische Fachschule für Chinesische Medizin in Zürich durchgeführt.

\section{Akupunkturnadeln}

Eingesetzt wurden sterilisierte Einweg-Akupunkturnadeln mit zylinderförmigem Schaft aus materialgleichem Spiraldraht sowie Führungsröhrchen des Herstellers Haeng Lim Seo Won, Seoul, Korea. Die Nadelabmessung inklusive Schaft betrug $0,30 \times 59,8 \mathrm{~mm}$. Die Polyethylen-Führungsröhrchen $(0,18 / 0,28 \times 55 \mathrm{~mm})$ wurden im Versuch auf $46,8 \mathrm{~mm}$ gekürzt.

\section{HRV-Messgerät}

Zur Aufzeichnung des Elektrokardiogramms wurde ein Prototyp des UBW (Universal Body Wave) Mobile Phone vom I.M.I. Ltd. - Swiss Independent Institute for Medical Innovation, Wanchai, Hong Kong, China verwendet. Bei diesem Gerät handelt es sich um ein handelsübliches Mobiltelefon, welches hardwareseitig mit drei elektronischen und einer optischen Diode sowie softwareseitig mit einem Messprogramm aufgerüstet wurde. Messperiodendauer: 140 s, Abtastrate: $500 \mathrm{~Hz}$, Störsignalschwelle: $100 \mathrm{~dB}$, Auflösung <2.7 mV/bit bei 19 bit, Messfehlertoleranz: 97\%. Das Gerät wurde bei vergleichbaren Studien eingesetzt $[15,16]$.

\section{Probanden}

Die Probanden waren im Alter zwischen 23 und 56 Jahren (27 Frauen und 3 Männer). Es handelte sich um Studierende und Personal der Tao Chi, Schweizerische Fachschule für Chinesische Medizin in Zürich. Ausschlusskriterien waren Herzerkrankungen, Einnahme von Medikamenten mit Einfluss auf Herz oder Nervensystem, Diabetes mellitus, Schilddrüsenerkrankungen, Hypertonie, Herzschrittmacher und sichtbar fortgeschrittene Schwangerschaft. Die Probanden meldeten sich freiwillig für den Versuch und unterzeichneten eine Einverständniserklärung. Die zuständige Ethikkommission (Ethikkommission des Kantons Zürich) äußerte keine Bedenken gegenüber dem Versuch.

\section{Therapeut}

Der Therapeut (R.J.H.) verfügt über mehr als 10 Jahre praktische Erfahrung sowie über eine fundierte Ausbildung in Chinesischer Medizin und Akupunktur von mehr als $3500 \mathrm{~h}$. Zum Zeitpunkt der Studie dozierte er an der Tao Chi, Schweizerische Fachschule für Chinesische Medizin in Zürich. Sämtliche Versuchsschritte wie Instruktion und Randomisierung, Stimulation der Nadel etc. wurden von ihm durchgeführt.

\section{Endpunkte}

In Tabelle 1 werden die in dieser Studie berücksichtigten Endpunkte bzw. Parameter aufgeführt.

Statistische Auswertung

Die Daten wurden anonymisiert und in IBM SPSS (Version 21, Armonk, NY, USA) statistisch ausgewertet. Da die Werte und auch die Differenzen der Variablen zwischen Baselinemessung und Endmessung nicht normalverteilt waren, wurden der Wilcoxon-Test und der Friedman-Test verwendet. $\mathrm{P}<0,05$ wurde als statistisch signifikant betrachtet.

\section{Ergebnisse}

Insgesamt konnten Daten von 24 Probanden à 3 Treffen ausgewertet werden (Tab. 2). Bei fünf Probanden war die HRV-Messung aus technischen Gründen nicht möglich; eine Probandin erschien aus persönlichen Gründen nicht zum dritten Treffen.

Die Herzfrequenz sank zwischen der Baseline und der Endmessung bei der Nullintervention tendenziell (Effektgröße und 95\%-Konfidenzintervall (KI): $\mathrm{d}=0,13(-0,44,0,69))$ und bei der Prüfintervention $(\mathrm{d}=0,24(-0,33,0,81))$ und der Kontrollintervention $(\mathrm{d}=0,24(-0.33,0,80))$ signifikant ab. Bei der Prüfintervention erhöhte sich die RMSSD (root mean square of successive differences) signifikant $(\mathrm{d}=-0,32(-0,88,0,26))$ gegenüber der Baselinemessung. Zwischen den drei Interventionen fanden sich keine signifikanten Unterschiede. 
Tab. 2. Ergebnisse der Messungen zur Herzratenvariabilität $(\mathrm{HRV} ; \mathrm{N}=24)$

\begin{tabular}{|c|c|c|c|c|}
\hline Parameter & Intervention & $\begin{array}{l}\text { Baselinemessung, } \\
\mathrm{MW} \pm \mathrm{SD}\end{array}$ & $\begin{array}{l}\text { Endmessung, } \\
\mathrm{MW} \pm \mathrm{SD}\end{array}$ & p-Wert \\
\hline \multirow[t]{3}{*}{ Bpm } & Nullintervention & $69,8 \pm 9,8$ & $68,5 \pm 9,1$ & 0,070 \\
\hline & Prüfintervention & $66,0 \pm 8,2$ & $64,0 \pm 7,6$ & $0,005^{*}$ \\
\hline & Kontrollintervention & $66,4 \pm 8,1$ & $64,4 \pm 8,2$ & $0,015^{\star}$ \\
\hline \multirow[t]{3}{*}{$\mathrm{LF}, \mathrm{ms}^{2}$} & Nullintervention & $951,7 \pm 972,5$ & $983,6 \pm 881,6$ & 0,587 \\
\hline & Prüfintervention & $1097,6 \pm 971,4$ & $1288,3 \pm 1418,8$ & 0,977 \\
\hline & Kontrollintervention & $1117,2 \pm 1371,2$ & $1399,7 \pm 1794,9$ & 0,265 \\
\hline \multirow[t]{3}{*}{$\mathrm{HF}, \mathrm{ms}^{2}$} & Nullintervention & $879,3 \pm 842,8$ & $859,6 \pm 927,7$ & 0,775 \\
\hline & Prüfintervention & $973,8 \pm 752,3$ & $1145,2 \pm 1184,5$ & 0,278 \\
\hline & Kontrollintervention & $1039,5 \pm 1165,0$ & $1297,9 \pm 1351,2$ & 0,568 \\
\hline \multirow[t]{3}{*}{ LF:HF } & Nullintervention & $2,96 \pm 5,36$ & $2,00 \pm 2,78$ & 0,909 \\
\hline & Prüfintervention & $3,14 \pm 5,10$ & $2,12 \pm 2,58$ & 0,407 \\
\hline & Kontrollintervention & $2,23 \pm 3,69$ & $2,14 \pm 4,25$ & 0,549 \\
\hline \multirow[t]{3}{*}{ SDNN, ms } & Nullintervention & $61,4 \pm 26,6$ & $67,0 \pm 37,2$ & 0,689 \\
\hline & Prüfintervention & $68,2 \pm 36,1$ & $80,3 \pm 49,0$ & 0,219 \\
\hline & Kontrollintervention & $70,4 \pm 38,0$ & $70,3 \pm 33,4$ & 0,627 \\
\hline \multirow[t]{3}{*}{ RMSSD, ms } & Nullintervention & $52,8 \pm 25,2$ & $55,3 \pm 26,1$ & 0,627 \\
\hline & Prüfintervention & $57,5 \pm 28,1$ & $67,2 \pm 33,4$ & $0,043^{*}$ \\
\hline & Kontrollintervention & $64,9 \pm 36,1$ & $67,6 \pm 35,4$ & 0,808 \\
\hline
\end{tabular}

*Signifikante p-Werte $(<0,05), \mathrm{MW}=$ Mittelwerte; SD = Standardabweichung.

$\mathrm{Bpm}=$ beats per minute (Schläge pro Minute); LF = low frequency power (niedrige Frequenzanteile); HF = high frequency power (hohe Frequenzanteile); SDNN = standard deviation of normal-to-normal (Standardabweichung normal bis normal (der RR-Intervalle)); RMSSD = root mean square of successive difference (quadratischer Mittelwert der aufeinanderfolgenden Differenzen).

\section{Diskussion und Schlussfolgerung}

In einem kürzlich publizierten Review zu Akupunktur und HRV [1] zeigten 14 Studien homogene Resultate, mit einer durchgehenden Erhöhung der hohen Frequenzanteile (HF) und einer Senkung des Verhältnisses zwischen niedrigen- und hohen Frequenzanteilen (LF:HF), sowohl bei Kranken wie auch bei Gesunden. Doch nicht bei allen Studien kam es zu den gleichen Resultaten. So führte beispielsweise die Untersuchung mit Akupunktur bei gesunden Probanden an Pe6 und einem benachbarten Sham-Punkt zu keinen messbaren Veränderungen der LF, der HF oder des LF:HF [21]. Bei einer weiteren Untersuchung an gesunden Probanden bewirkte Akupunktur und Sham-Akupunktur an Pe6 die Erhöhung des durchschnittlichen RR-Intervalls, aber nur bei VerumAkupunktur konnte eine Erhöhung der normalisierten HF und der Standardabweichung des RR-Intervalls festgestellt werden

Während mit Laser-Akupunktur (685 nm und 880-950 nm) an Pe6 bei gesunden Männern keine Veränderungen der HRV-Parameter ermittelt werden konnten [22], wurde bei einem Versuch mit blauem Laserlicht (405 nm) in der Verumgrupppe eine gesenkte Herzfrequenzrate gemessen, nicht aber in der Kontrollgruppe, wo der Laser deaktiviert war [23]. Da die Laser-Akupunktur keine mechanische Komponente aufweist, könnte sie über andere Mechanismen wirken, wodurch das Ausbleiben von Veränderungen der HRV-Parameter aber nicht vollständig erklärt werden kann.

In einer Studie zu HRV und Elektrogastrographie wurden Pe6 und Ma36 einmal mit und einmal ohne Stimulation akupunktiert [24]. Die Stimulation der Akupunkturnadeln durch Rotation führte zu einer Veränderung der Anteile von Normo-, Brady- und Tachygastrie im Elektrogastrogramm. Von den gemessenen HRVParametern erhöhte sich durch die Akupunktur mit Stimulation ausschließlich die RMSSD. Daraus folgerten die Autoren der Studie, dass es sich bei der Nadelstimulation um einen wichtigen Faktor in der Akupunktur handle.

Ein japanisches Forscherteam verglich den Effekt der benachbarten Akupunkturpunkte Ren16 und Ren17 [25]. Die Stimulation von Ren16 zeigte keinen messbaren Effekt auf die HRV, die Stimulation von Ren 17 bewirkte hingegen eine signifikante Veränderung von Puls, HF und LF. Es ist anzumerken, dass für diese Studie minimalinvasiv akupunktiert wurde, also mit ca. 20 g Insertionsdruck und sofortiger Entfernung der Nadel ca. $1 \mathrm{~s}$ nach Erreichen einer Einstichtiefe von 5-7 mm. Die Nadeln wurden nicht stimuliert, es wurde kein De Qi (die vom Patienten oder Therapeuten wahrgenommene 'energetische' Empfindung $[12,26])$ provoziert, und der therapeutische Kontakt wurde auf das Mindestmaß reduziert.

Es ist ungeklärt, weshalb in einigen Studien der Einfluss von Akupunktur an Pe6 auf die HRV ermittelt werden konnte und in anderen nicht. Ein Grund könnte sein, dass Akupunktur regulierend wirkt. So reagieren beispielsweise Kranke anders auf Akupunktur als Gesunde, wie unter anderem in Studien mit funktioneller Magnetresonanztomographie gezeigt werden konnte [27].

Unter den hier gewählten Bedingungen konnten wir den zuvor subjektiv wahrgenommenen Unterschied zwischen Stimulation mit 'therapeutischem Qi' (durch Berührung, aber ohne mechanische Bewegung der Nadel) und keiner Stimulation anhand von objektiven HRV-Parametern nicht eindeutig bestätigen. Allerdings war die Interventionsdauer mit 10 min eher kurz, im Verhältnis zu 
vergleichbaren Studien, in denen 20-30 min mit Akupunktur interveniert wurde [1]. Außerdem vermuten wir, dass anstelle der hier punktuell (vorher/nachher) durchgeführten Messung ein durchgehendes Elektrokardiogramm aussagekräftigere Daten zur Ermittlung von Veränderungen der HRV ergeben würde.

\section{Dank}

Wir danken der Schweizerischen Berufsorganization für Traditionelle Chinesische Medizin (SBO-TCM) sowie Ann St. Clair Renard für die finanzielle Unterstützung zur Freischaltung (open access) dieser Publikation.

\section{Disclosure Statement}

Die Autoren erklären hiermit, dass keinerlei Interessenskonflikte in Bezug auf dieses Manuskript bestehen.

\section{Literatur}

1 Chung JW, Yan VC, Zhang H: Effect of acupuncture on heart rate variability: a systematic review. Evid Based Complement Alternat Med 2014;2014:819871.

2 Pumprla J, Howorka K, Groves D, et al.: Functional assessment of heart rate variability: physiological basis and practical applications. Int J Cardiol 2002;84:1-14.

3 Skoien J: Die Grundlagen der Traditionellen Chinesischen Medizin: Ein umfassendes Lern- und Arbeitsbuch für Praktiker und Studenten. Marburg, Tectum Verlag, 2005.

4 Unbekannte Autoren: Göttliche Angelpunkte des Gelben Kaisers' Klassiker des Innern (Chin. Orginaltitel: Huáng Dì Nèi Jing Líng Shū 黄 帝内经 灵枢). Ca. 1030 v.u.Z.- 24 n.u.Z.

5 Unbekannte Autoren: Grundlegende Fragen des Gelben Kaisers` Klassiker des Innern (Chin. Orginaltitel: Huáng Dì Nèi Jīng Sù Wèn 黄帝内经素問). Ca. 1030 v.u.Z.- 24 n.u.Z.

6 Johnson JA, Stewart JM, Howell MH: Chinese Medical Qigong Therapy: A Comprehensive Clinical Guide. Pacific Grove, International Institute of Medical Qigong, 2000.

7 Ho TJ, Christiani DC, Ma TC, et al.: Effect of Qigong on quality of life: a cross-sectional population-based comparison study in Taiwan. BMC Public Health 2011;11:546.

8 Chang MY: Qigong effects on heart rate variability and peripheral vasomotor responses. West J Nurs Res 2015;37:1383-403.

9 Yan X, Shen H, Jiang H, et al.: External Qi of Yan Xin Qigong inhibits activation of Akt, Erk1/2 and NF-kB and induces cell cycle arrest and apoptosis in colorecta cancer cells. Cell Physiol Biochem 2013;31:113-122.
10 Yan X, Li F, Dozmorov I, et al.: External Qi of Yan Xin Qigong induces cell death and gene expression alterations promoting apoptosis and inhibiting proliferation, migration and glucose metabolism in small-cell lung cancer cells. Mol Cell Biochem 2012;363:245-55.

11 Yan X, Shen H, Jiang H, et al.: External Qi of Yan Xin Qigong differentially regulates the Akt and extracellular signal-regulated kinase pathways and is cytotoxic to cancer cells but not to normal cells. Int J Biochem Cell Biol 2006;38:2102-113.

12 Chace C: The shape of Qi. Enhancing the vocabulary of contact in acupuncture. The Lantern 2008;5:4-11.

13 Hochstrasser RJ, Endler PC, Klein SD: Perception of therapeutic Qi, a nonmechanical, nonpsychological factor in acupuncture that originates from the Therapist. J Acupunct Meridian Stud 2015;8:203-208.

14 Huang ST, Chen GY, Lo HM, et al.: Increase in the vagal modulation by acupuncture at neiguan point in the healthy subjects. Am J Chin Med 2005;33:157-164.

15 Pizzinato E, Endler PC, Muller J, et al.: Heart rate variability in a study on reiki treatment. The Open Complementary Medicine Journal 2012;4:12-15.

16 Girsberger W, Banziger U, Lingg G, et al.: Heart rate variability and the influence of craniosacral therapy on autonomous nervous system regulation in persons with subjective discomforts: a pilot study. J Integr Med 2014;12:156-161.

17 Hochstrasser RJ, Endler PC, Klein SD: Introduction of two novel devices for investigating the influence of non-mechanical components such as therapeutic Qi in acupuncture. J Integr Med 2013;11:168-174.

18 No authors listed: Heart rate variability: standards of measurement, physiological interpretation and clinical use. Task Force of the European Society of Cardiology and the North American Society of Pacing and Electrophysiology. Circulation 1996;93:1043-1065.
19 Wittling W: Diagnostik der Herzratenvariabilität: Einblicke in die autonom-nervöse Regulation von Stressverarbeitung, Befindlichkeit, Verhalten und Gesundheit. Trier, ZNF, 2009.

20 Wittling W, Schweiger E: Auswirkungen einer frequenzmodulierten Magnetfeld- und Laserstimulation auf das autonome Nervensystem: Eine Validierungsstudie. Freiburg, ZNF Forschungsberichte, 2009.

21 Chang S, Chao WL, Chiang MJ, et al.: Effects of acupuncture at Neiguan (PC 6) of the pericardial meridian on blood pressure and heart rate variability. Chin J Physiol 2008;51:167-177.

22 Hubscher M, Vogt L, Banzer W: Laser needle acupuncture at Neiguan (PC6) does not mediate heart rate variability in young, healthy men. Photomed Laser Surg 2007;25:21-5.

23 Litscher G, Xie Z, Wang L, Gaischek I: Blue $405 \mathrm{~nm}$ laser light mediates heart rate - investigations at the acupoint Neiguan (Pe.6) in Chinese adults. N Am J Med Sci 2009;1:226-231.

24 Witt CM, Meissner K, Pach D, Thiele C, et al.: Stimulation of gastric slow waves with manual acupuncture at acupuncture points ST36 and PC6 - a randomized single blind controlled trial. Neurogastroenterol Motil 2012;24:438-445.

25 Kurono Y, Minagawa M, Ishigami T, et al.: Acupuncture to Danzhong but not to Zhongting increases the cardiac vagal component of heart rate variability. Auton Neurosci 2011;161:116-20.

26 Yang XY, Shi GX, Li QQ, et al.: Characterization of deqi sensation and acupuncture effect. Evid Based Complement Alternat Med 2013;2013:319734.

27 Huang W, Witt CM, Pach D: Characterizing acupuncture stimuli using brain imaging with 7MRI. A systematic review and meta-analysis of the literature. PLoS One 2012;7:e32960. 